\title{
AN OPERATION FOR CHRONIC PREPATELLAR BURSITIS
}

\author{
J. B. Quayle, London and M. P. Robinson, Crewe, England \\ From the Robert Jones and Agnes Hunt Orthopaedic Hospital, Oswestry, Shropshire, and \\ the Leighton Hospital, Crewe, Cheshire
}

\begin{abstract}
An operation for chronic prepatellar bursitis is described in which only the posterior wall of the bursa is excised, thus preserving, undamaged, healthy and normally sensitive skin. This procedure is easier and less traumatic than complete excision of the bursa and results in fewer complications. It is suggested that removal of the anterior wall of the bursa results in unnecessary and harmful interference with the underlying skin. The operation described gives a good functional and structural result; leaving the anterior wall of the bursa does not predispose to recurrence.
\end{abstract}

Prepatellar bursitis is a common condition and is usually due to recurrent trauma, although gout, sepsis and rheumatoid arthritis are other predisposing causes. In chronic traumatic bursitis the bursa undergoes irreversible pathological damage. Initially it is distended by a haemorrhagic effusion. Chronic inflammatory change takes place. The wall becomes thickened with collagen and the synovial lining atrophies, though parts proliferate into pseudovilli. Cholesterol crystals may form in fusiform clefts within the thickened wall as a result of fatty degeneration, and induce an infiltration of histiocytes and giant cells. Irregular deposition of fibrin occurs following attempts at organisation of the haematoma. Most cases are mild and become quiescent with conservative treatment. The effusion subsides, leaving the prepatellar area only slightly tender. The thickened granular consistency of the bursa on palpation reflects the histological changes described above. Bandaging, with avoidance of further trauma, is usually successful. Further unavoidable trauma, however, as in miners, farmers and over-conscientious housewives, results in a progressive enlargement of the bursa, which becomes even more vulnerable to injury.

Such patients require operation, and total excision of the bursa is often carried out. Unfortunately, we have found certain shortcomings with this method. The dissection itself is not always simple because the thick anterior wall of the inflamed bursa is adherent to the overlying skin, whose structure is damaged by the operation (Fig. 1). Not uncommonly the final outcome is an unpleasantly sensitive knee, with thin, atrophic skin adherent to the underlying patella; kneeling is then impossible. Postoperative interstitial and subcutaneous haematoma is not uncommon and can cause troublesome delayed healing. However, dissection in the relatively avascular plane

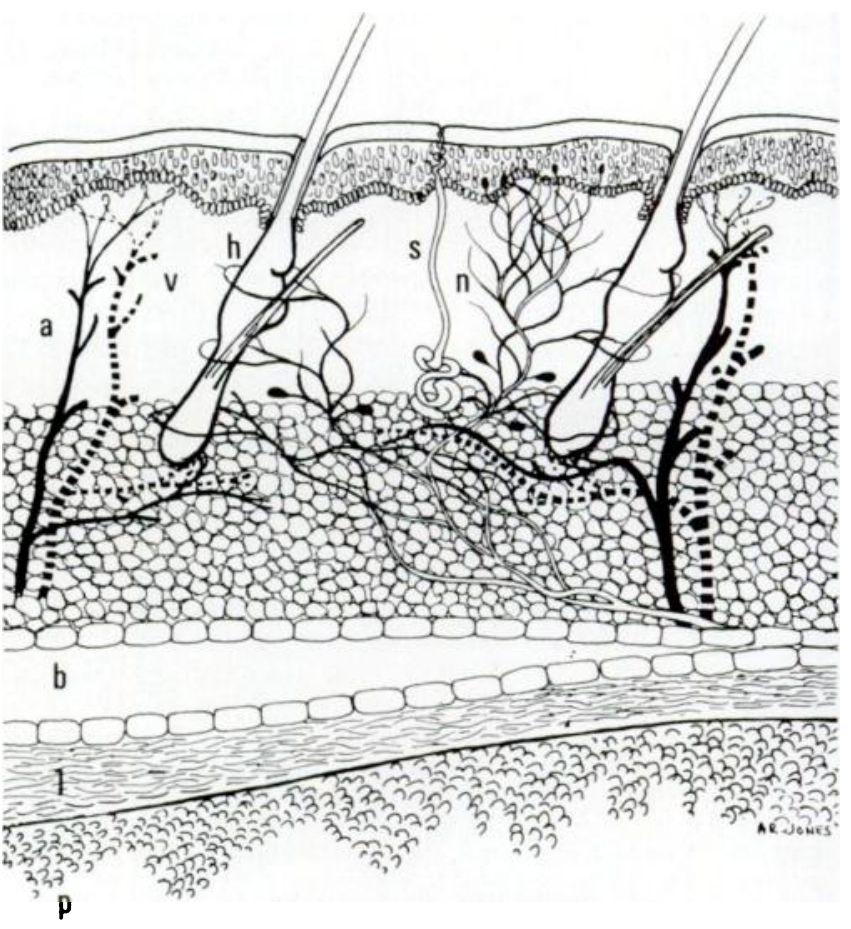

Fig. 1

Schematic section of skin, bursa and patella: a-artery, b-bursa, $\mathrm{h}$-hair follicle, 1 - loose areolar tissue, $n-$ nerve plexus, $\mathrm{p}$-patella, $s$-sweat gland, $v$-vein.

between the patella and the posterior wall of the bursa is comparatively easy, and much less traumatic. We felt 
that if we confined our dissection to this plane and removed only the posterior wall of the bursa, thereby leaving the anterior wall with its overlying skin undisturbed, the knee would be protected by healthy, undamaged, supported skin, and the chronic bursitis also cured.

\section{ANATOMY AND TECHNIQUE}

The prepatellar bursa is a subcutaneous synovial bursa which lies in front of the patella and facilitates movement of the skin over this bone. It may cover a wide area and
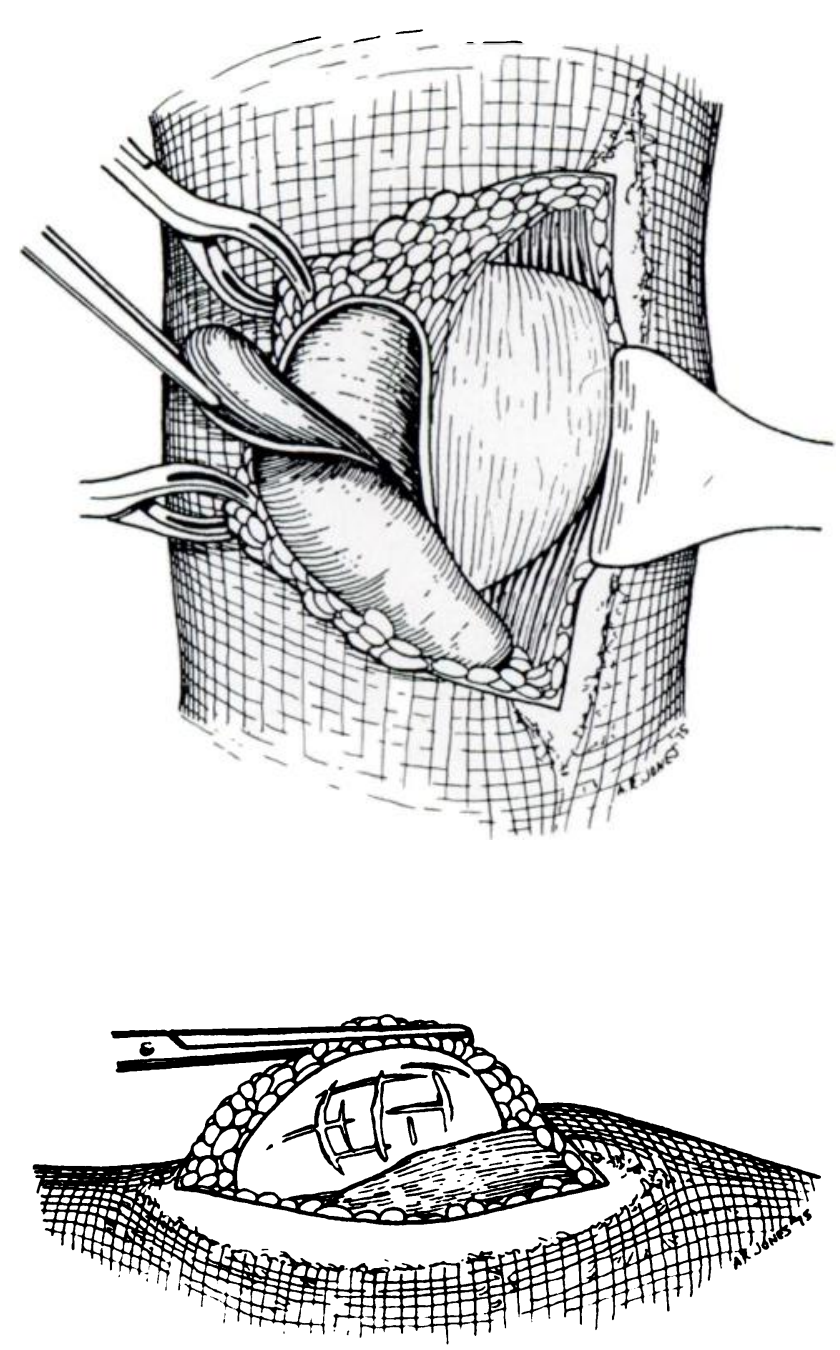

FIG. 3

Figure 2-Excision of the posterior wall of the prepatellar bursa. Figure 3-Scarification of anterior wall of the bursa.

even blend with the subcutaneous infrapatellar bursa over the tibial tubercle.

Operation-A medial curved parapatellar incision is made and deepened to the extensor expansion. If the bursa is large, a lateral incision may be better, thus avoiding the infrapatellar branch of the saphenous nerve. The flap, which includes the prepatellar bursa, is turned back laterally and the posterior wall of the bursa is excised (Fig. 2). The lining of the anterior wall and the anterior aspect of the patella is then scarified (Fig. 3) and the flap laid back over the patella and resutured. A compression bandage is applied and retained for ten days, when the sutures are removed.

\section{MATERIAL}

Eight patients who had this operation have been reviewed and the results are shown in Table 1 . All had had chronic prepatellar bursitis for longer than twelve months, two patients for more than two years. The initiating factor was usually trauma of only moderate intensity, and infection did not appear to be a contributory cause, or later complication. There was a preponderance of women in the group (five to three) and the occupations of most patients involved excessive kneeling, which eventually became impossible. We considered that the activities of the housewives in the group were indeed more arduous than usual, particularly since they also performed parttime labouring jobs. Underlying disease was excluded by radiographs and by screening tests for tuberculosis, syphilis, brucellosis, gout and rheumatoid arthritis, and later by review of the histology of excised tissue. In fact, suspicion of pre-existing pathology was found in only one case (Case 6) in which histology of the bursa was suggestive of early rheumatoid arthritis. In all patients the original swelling never subsided completely, but became gradually larger and more troublesome, and was aggravated by repeated mild trauma, mainly kneeling. Curative aspirations were attempted in four patients without success; no sclerosant fluids were introduced.

In the review, particular interest was focused on assessing the structure and function of the knee after operation, comparison being made with the unaffected normal knee. Attention was directed to determining the quality and thickness of the skin; the degree of skin tethering; the laxity of skin over the patella; sensation over the knee to touch and pressure; and whether the operated side was used for kneeling as much as the normal side.

\section{RESULTS}

No operative difficulties were experienced. One patient (Case 1) developed a small haematoma which subsided spontaneously. The incisions in two patients (Cases 4 and 7) with large bursae were carried too far distally and damaged the infrapatellar branch of the saphenous nerve. After initial reluctance, they now kneel normally but very slight hypoaesthesia has persisted for two years. Two patients admitted some mild irritation and discomfort in the scar itself. Unlike the others, their scars tended to be tethered to the underlying extensor expansion. The quality of skin overlying the patella in each patient 
TABLE I

Clinical. Detall.s (Eight Cases)

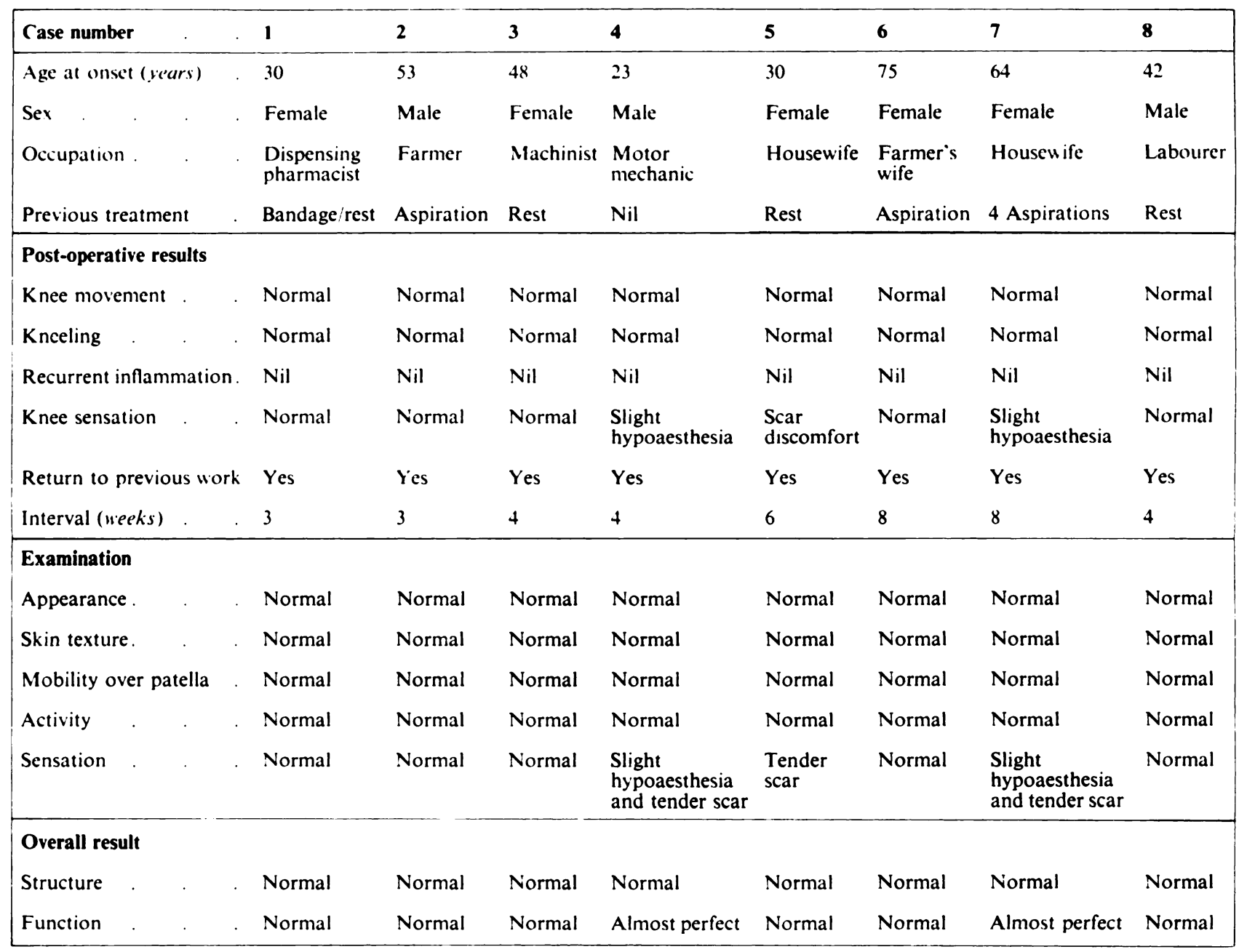

appeared and felt normal, and was as lax and mobile as kneeling. Sensation to touch and pressure was normal in that on the unoperated normal knee. All patients had all patients, except the two described. On average, four returned to their previous activities within six weeks and, years have passed since operation, the earliest being eight after initial apprehension, experienced no difficulty in years ago, and no patient has had further trouble.

We wish to express our gratitude and thanks to Mr Alan Jones, Educational Technology Unit at St George's Hospital, for his help with the diagrams. 